\title{
Interações medicamentosas entre terapias anti-hipertensivas e terapêutica odontológica: revisão de literatura
}

\author{
Drug interactions among antihypertensive agents and dental drugs: a literature review
}

\author{
Saulo Rodrigues dos $\operatorname{Santos}^{1^{\bullet}}$, André Luis Silva Santos ${ }^{\bullet}$, Hélio Igor Melo de Albuquerque ${ }^{\bullet}$, Luiz \\ Carlos Ferreira da Silva ${ }^{3}$
}

${ }^{1}$ Departamento de Odontologia da Universidade Federal de Sergipe, Aracaju, Sergipe, Brasil. ${ }^{2}$ Cirurgião Buco-Maxilo-Facial e Mestre em
Odontologia pela Universidade Federal de Sergipe, Sergipe, Brasil. ${ }^{3}$ Professor Associado do Departamento de Odontologia da
Universidade Federal de Sergipe, Aracaju, Sergipe, Brasil. *Autor para correspondência. E-mail: saulorodrigues2@academico.ufs.br

Resumo: Introdução: A hipertensão arterial afeta boa parte da população brasileira, sendo um quadro comumente encontrado na população masculina e em idosos, necessitando, por muitas vezes, do emprego de fármacos anti-hipertensivos. Interações medicamentosas podem ocorrer nos pacientes hipertensos que estão em tratamento odontológico, com risco real de efeitos adversos e danos permanentes. Esta revisão de literatura tem como objetivo esclarecer sobre as interações medicamentosas que podem ocorrer entre as terapias anti-hipertensivas e aquelas adotadas no desenvolvimento dos tratamentos odontológicos, para que se aumente a segurança das prescrições aos pacientes. Revisão: A metodologia empregada neste trabalho envolveu o levantamento bibliográfico nas bases de dados Bireme e Lilacs, no indexador Scielo, além do Google acadêmico e das obras literárias disponíveis acerca do tema. A coleta de dados compreendeu artigos publicados nos últimos 12 anos, período de 2009 a 2021. Discussão: Dentre as medicações empregadas na rotina odontológica, os anti-inflamatórios não esteroidais, os AINES, chamam atenção pela grande ocorrência de interações junto às classes medicamentosas preconizadas no tratamento contra a hipertensão, observando-se, sobretudo, a diminuição dos efeitos desejáveis para o controle da pressão arterial. Considerações finais: Tendo em vista que os riscos das interações são iminentes na Odontologia, a prescrição e a aplicação rotineira dos fármacos devem ser feitas de forma segura e responsável, levando em consideração todo o histórico médico do paciente hipertenso, assim como pela necessidade de se conhecer os riscos das associações entre os medicamentos por parte dos profissionais e acadêmicos.

Palavras-chave: interações medicamentosas, hipertensão, anti-hipertensivos, tratamento odontológico.

\begin{abstract}
Introduction: Arterial hypertension affects a huge part of the Brazilian population and this condition is commonly found within the male and elderly population, leading to the use of antihypertensive medication in some cases. Drug interactions may happen in hypertensive patients undergoing dental treatment with real risks of side effects and permanent damage. This literature review aims to clarify the subject of drug interactions that may happen among antihypertensive agents and those implemented in dental treatments in order to increase safety toward patient prescriptions. Review: The methodology involved and extensive bibliographical research on Bireme and Lilacs databases, Scielo indexer, Google Scholar, and literary work available on the subject. The data collection included papers published in the last 12 years, from 2009 to 2021. Discussion: Among the most commonly used medications in routine dental care, the Nonsteroidal Anti-Inflammatory Drugs (NSAIDs) resulted as the most noteworthy aspect of this research due to the enormous incidence of interactions when associated with anti-hypertensive drugs, especially decreasing the expected effects of such drugs in regulating blood pressure. Final considerations: Once the risks of drug interactions in Dentistry are imminent, the routine drug prescription and application must be performed safely and responsibly by professionals and students alike, taking into account the hypertensive patient's full medical history and understanding the risks that the drug associations may cause.
\end{abstract}

Keywords: drug interactions, hypertension, antihypertensive agents, dental care.

\section{Introdução}

A hipertensão arterial (HA) é uma doença crônica não transmissível que engloba múltiplas condições associadas dependentes de fatores genéticos, sociais e ambientais. Com frequência, encontrada de forma assintomática, podendo promover modificações nas estruturas e/ou em órgãos como o cérebro, coração e rins. Seu controle se dá tanto por meios não farmacológicos, tendo-se as mudanças de hábitos, a exemplo dos 
alimentares e estilo de vida, bem como por meios farmacológicos, quando se faz o emprego de medicamentos que promovem ação anti-hipertensiva (Barroso et al., 2021).

Na população brasileira, cerca de 32,3\% das pessoas fazem o uso de terapia anti-hipertensiva e têm pressão arterial (PA) maior ou igual a 140/90 mmHg. É uma condição mais comum nos homens, tendo-se prevalência de $71,7 \%$ para os pacientes acima dos 70 anos. Tem como fatores de risco, por exemplo, o consumo excessivo de sal, a obesidade, o sedentarismo e a elevação do colesterol e triglicerídeos (dislipidemia), tendo-se também associação com a diabetes, distúrbios metabólicos e mudanças funcionais e/ou estruturais dos órgãos que atuam na pressão arterial. O seu desenvolvimento pode estar ligado a eventos como o infarto agudo do miocárdio, insuficiência cardíaca, acidente vascular encefálico e a doença renal crônica (Barroso et al., 2021).

Cabe ao Cirurgião-Dentista, antes de qualquer procedimento, fazer o levantamento do histórico médico dos pacientes, sobretudo, para a identificação da condição de hipertensão, tanto pelo relato da comorbidade, do uso das terapias anti-hipertensivas e pela aferição da PA. Tais dados são importantes para a verificação dos prováveis riscos que podem vir a ser acarretados frente aos tratamentos odontológicos, especialmente, pela possibilidade das interações medicamentosas que podem ocorrer entre as medicações do controle da PA e aquelas empregadas na rotina odontológica (Little et al., 2009).

Sem dúvida, o estabelecimento do histórico médico, sobretudo no levantamento sobre as medicações utilizadas pelo hipertenso, ajuda na hora de estabelecer possíveis riscos. O tratamento farmacológico antihipertensivo, na maioria dos casos, inicia-se com uma medicação, sendo incluídos outros fármacos posteriormente. Dentre as classes medicamentosas anti-hipertensivas, algumas delas podem intervir no sistema renina-angiotensina-aldosterona, a exemplo dos inibidores da enzima conversora de angiotensina, ou até mesmo na diminuição da resistência vascular periférica por meio do estímulo da diurese, como ocorre com os diuréticos (Haese \& Cançado, 2016; Cavalcante, 2018; Barroso et al., 2021).

As interações medicamentosas devem estar no entendimento dos futuros profissionais da Odontologia. Elas são caracterizadas como as modificações na farmacocinética e farmacodinâmica devido à interação de uma droga com outra substância. Vários fatores contribuem para essa ocorrência, tal como os pacientes ditos polifarmácia, principalmente em idosos acima dos 65 anos, seja pelo uso de medicamentos sem prescrição médica ou até mesmo as interações com produtos naturais com fins terapêuticos de uso rotineiro, como os chás de ervas. A inclusão inadequada das classes medicamentosas comumente prescritas ou aplicadas nos procedimentos odontológicos, sem a observância e entendimento das drogas de uso crônico ou prévio destinadas aos pacientes perante o quadro hipertensivo, pode acarretar em efeitos adversos prejudiciais (Bertollo et al., 2013; Nascimento et al., 2017).

\section{Revisão}

Para realização da revisão sistemática da literatura acerca do tema, foram utilizadas obras literárias que continham relação com as temáticas relevantes para este trabalho. As bases de dados empregadas para o rastreamento dos artigos científicos foram Bireme e Lilacs; o indexador Scielo, além do Google Acadêmico. Utilizou-se na busca os seguintes descritores: interações medicamentosas (drug interactions), hipertensão (hypertension), anti-hipertensivos (antihypertensive agents) e tratamento odontológico (dental care). Foram considerados apenas artigos em português, publicados em periódicos do Brasil. A coleta de dados compreendeu artigos publicados nos últimos 12 anos, período de 2009 a 2021. Todos os resumos dos artigos contendo as palavras-chaves foram lidos e avaliados, sendo posteriormente selecionados aqueles que abrangiam questões sobre interações medicamentosas entre terapias anti-hipertensivas e tratamentos empregados na Odontologia. O rastreamento identificou 39 artigos, dos quais, apenas 12 se enquadraram nos critérios da pesquisa.

\section{Discussão}

\section{Hipertensão Arterial}

A hipertensão arterial (HA) é caracterizada pelos níveis de pressão acima de $140 \mathrm{mmHg}$ para a pressão arterial sistólica (PAS) e/ou $90 \mathrm{mmHg}$ para a pressão arterial diastólica (PAD) (Barroso et al., 2021). Existem diversos meios para o controle endógeno da pressão arterial, a exemplo dos reflexos nervosos autônomos e da moderação pressórica desempenhada pelos rins, seja pela produção de hormônios ou pela regulação do volume sanguíneo (Hall, 2017). 
A HA pode ser primária ou secundária. A primária é a predominante, sendo que o seu controle se dá pela mudança dos hábitos, a exemplo do controle da ingestão de sal e gorduras, na prática de exercícios físicos e na suspensão do hábito tabagista, por exemplo. Quando a compensação da pressão arterial não é alcançada pelos meios anteriormente mencionados, medicações são prescritas aos pacientes, como os betabloqueadores e inibidores da enzima conversora de angiotensina. A hipertensão secundária é consequência de outras doenças, como o hipertireoidismo e as disfunções renais (Andrade, 2014). Além disso, tem-se hipertensão do avental branco, a qual é o aumento da pressão arterial quando o paciente está na presença dos profissionais da saúde, sendo de suma importância que o Cirurgião-Dentista saiba da sua existência. Esta condição ocorre principalmente em mulheres com idades mais avançadas (Matos et al., 2018).

A pressão arterial e a hipertensão são classificadas de acordo com as medidas encontradas na aferição. Para os adultos as referências para a PA encontram-se no quadro abaixo:

Quadro 1. Adaptação dos valores das pressões arteriais sistólica e diastólica para adultos da $7^{\text {a }}$ Diretriz Brasileira de Hipertensão Arterial (SBC, 2016) e das Diretrizes Brasileiras de Hipertensão Arterial 2020 (Barroso et al., 2021).

\begin{tabular}{|c|c|c|}
\hline Classificação & Pressão Arterial Sistólica - PAS & Pressão Arterial Diastólica - PAD \\
\hline Pressão ótima & $<120 \mathrm{mmHg}$ & $<80 \mathrm{mmHg}$ \\
\hline Pressão normal & $120-129 \mathrm{mmHg}$ & $80-84 \mathrm{mmHg}$ \\
\hline Pré-hipertensão & $130-139 \mathrm{mmHg}$ & $85-89 \mathrm{mmHg}$ \\
\hline Hipertensão estágio 1 & $140-159 \mathrm{mmHg}$ & $90-99 \mathrm{mmHg}$ \\
\hline Hipertensão estágio 2 & $160-179 \mathrm{mmHg}$ & $100-109 \mathrm{mmHg}$ \\
\hline Hipertensão estágio 3 & $\geqslant 180 \mathrm{mmHg}$ & $\geqslant 110 \mathrm{mmHg}$ \\
\hline Hipertensão Sistólica Isolada & $\geqslant 140 \mathrm{mmHg}$ & $<90 \mathrm{mmHg}$ \\
\hline
\end{tabular}

\section{Classes medicamentosas empregadas no controle da hipertensão arterial}

A maioria dos pacientes hipertensos necessita da terapia medicamentosa associada a mudança de hábitos para que tenha o controle da pressão arterial. O esquema terapêutico pode ser a monoterapia, sendo a primeira conduta anti-hipertensiva de eleição, tendo-se como classes medicamentosas preferenciais os diuréticos tiazídicos, os bloqueadores de canais de cálcio, os inibidores da enzima conversora de angiotensina e os bloqueadores dos receptores da angiotensina II. Para grande parte dos pacientes, o controle da hipertensão se dá pela combinação de fármacos anti-hipertensivos, tendo-se, inicialmente, a combinação de dois medicamentos com a inclusão de outros ao longo do tempo até que a PA seja controlada (Ritter et al., 2020; Barroso et al., 2021).

\section{Diuréticos Tiazídicos (Clortalidona, Hidroclorotiazida e Indapamida)}

São os diuréticos preferenciais no tratamento anti-hipertensivo. São medicamentos natriuréticos, os quais diminuem o volume extracelular e, por consequência, a resistência vascular periférica. É importante salientar que é comum fazer o uso dos diuréticos tiazídicos em conjunto com os poupadores de potássio. Ligam-se ao sítio do cloro no cotransportador $\mathrm{Na}+/ \mathrm{Cl}$ - no túbulo distal, promovendo a inibição de sua ação, ocasionando natriurese (perda de sódio e cloreto pela urina), tendo-se o aumento da diurese (Ritter et al., 2020).

\section{Diuréticos de Alça (Furosemida e Bumetanida)}

São utilizados nos casos de insuficiência renal e quando há o desenvolvimento de edema. É comum a associação com os poupadores de potássio. São os diuréticos mais potentes, atuando sobre o ramo ascendente espesso da alça de Henle, promovendo a inibição do transportador Na+/K+/2CL-, ligando-se ao sítio do CL-, ocasionando a maior eliminação eletrolítica pelos rins, o que reflete na maior taxa diurética (Ritter et al., 2020; Barroso et al., 2021).

\section{Poupadores de Potássio (Espironolactona e Amilorida)}

Seu uso se dá na associação com os diuréticos de alça e tiazídicos. A espironolactona é um antagonista competitivo da aldosterona, haja vista que a competição pelos receptores intracelulares da aldosterona gera a inibição da retenção de $\mathrm{Na}+$ e a secreção de $\mathrm{K}+$. Já a amilorida atua sobre os túbulos e ductos coletores inibindo a reabsorção de $\mathrm{Na}+$ pelo bloqueio dos canais de sódio e diminuem a eliminação de $\mathrm{K}+$ (SBC, 2016; Ritter et al., 2020). 


\section{Agentes alfa-agonistas de ação central (Metildopa, Clonidina, Guanabenzo, Moxonidina e Rilmenidina)}

Agem na diminuição da atividade simpática e do barorreflexo, tendo-se como principais ações a bradicardia e o efeito da hipotensão que é vista no ortostatismo (hipotensão ortostática). A queda na pressão se dá pela inibição da liberação de norepinefrina, sobretudo, a metildopa (está caindo em desuso, sendo utilizada na gravidez), a qual origina o falso mediador metilnorepinefrina (Ritter et al., 2020; Barroso et al., 2021).

\section{Alfa bloqueadores (Doxazosina, Prazosina e Terazosina)}

São competidores seletivos dos receptores alfa-1, causando vasodilatação e queda da pressão arterial. Não aumentam a liberação de norepinefrina pelas terminações simpáticas, ocasionando menos eventos de taquicardia. Desta forma, esta classe leva a diminuição da resistência vascular periférica sem interferir no débito cardíaco. Também são usados no tratamento da hipertrofia prostática benigna, diminuindo assim a retenção urinária por ocorrer o relaxamento da musculatura lisa (Ritter et al., 2020; Barroso et al., 2021).

\section{Vasodilatadores diretos (Minoxidil e Hidralazina)}

A ação se dá no relaxamento da musculatura lisa arterial, diminuindo a resistência vascular periférica. Este efeito ocorre pela indução da abertura dos canais de potássio hiperpolarizando a célula, resultando na desativação dos canais de cálcio voltagem-dependentes (Ritter et al., 2020; Barroso et al., 2021).

Bloqueadores de canais de cálcio (os di-idropiridínicos - Anlodipino, Felodipino, Isradipino, Manidipino, Nifedipino, Nimodipino, Nitrendipino, Lercanidipino, Levanlodipino e Lacidipino; e os não di-idropiridínicos - Verapamila e Diltiazem)

Impedem o fluxo de cálcio para o meio intracelular, incluindo as células das musculaturas cardíaca e lisa das arteríolas, promovendo um bloqueio competitivo com o cálcio pelos canais voltagem-dependentes. Desta forma, a excitabilidade do coração e a frequência cardíaca são reduzidas. Tem ações desejáveis como a vasodilatação, redução da atividade contrátil do miocárdio e da condução atrioventricular. A resistência vascular periférica é reduzida, diminuindo a pressão arterial (Bombig \& Póvoa, 2009; SBC, 2016; Ritter et al., 2020; Barroso et al., 2021).

\section{Inibidores da enzima conversora de angiotensina - IECA - (Captopril, Enalapril, Ramipril, Lisinopril e Trandolapril)}

A ECA - enzima conversora de angiotensina - converte angiotensina I em II, tendo assim a ação de vasoconstrição e de reabsorção de sódio tubular, promovendo a maior retenção de líquidos, elevando a pressão arterial. Os fármacos IECA atuam em leitos capilares sensíveis à angiotensina, a exemplo dos rins, promovendo o efeito oposto do que é visto pela ação da ECA. São anti-hipertensivos eficazes, reduzindo a morbimortalidade. Porém, existem efeitos adversos no seu uso, a exemplo da hipotensão arterial sintomática, tosse seca e disfunção renal (SBC, 2016; Ritter et al., 2020; Barroso et al., 2021).

\section{Bloqueadores dos receptores AT1 da angiotensina II (Losartana e Valsartana)}

Os receptores AT1 quando ativados promovem ações vasoconstritoras, proliferativas e estimuladoras da liberação de aldosterona, influenciando, sobretudo, no aumento da resistência vascular periférica, havendo assim a elevação da pressão arterial. Os bloqueadores dos receptores de angiotensina II têm ação semelhante aos IECA. Bloquear tais receptores é importante pelo fato de a ECA não ser a única enzima capaz de converter angiotensina, tendo-se também a ação das quimases no desempenho dessa função, sendo que estas enzimas não sofrem a ação do IECA (SBC, 2016; Ritter et al., 2020; Barroso et al., 2021).

\section{Betabloqueadores (Caverdilol, Metoprolol, Nadolol, Nebivolol, Pindolol, Propranolol)}

São antagonistas dos receptores beta-adrenérgicos. Tem efeito vasodilatador, assim como diminui o débito cardíaco e reduz a liberação de renina pelas células justagromerulares dos rins. Além disso, promove a 
diminuição das catecolaminas nas sinapses nervosas. Os efeitos são benéficos para os tratamentos da hipertensão arterial e da insuficiência cardíaca (Scolari et al., 2018; Ritter et al., 2020; Barroso et al., 2021).

\section{Inibidores diretos da renina (Alisquireno)}

Diminui a ação da renina, resultando na menor liberação de angiotensina II (Barroso et al., 2021).

\section{Interações medicamentosa entre a terapia do paciente hipertenso e as medicações empregadas no tratamento odontológico}

As principais classes medicamentosas de uso sistêmico prescritas na Odontologia são os anti-inflamatórios não esteroidais (AINES), os antimicrobianos do tipo antibacterianos e os analgésicos não opioides. Além disso, anestésicos locais são bastante utilizados nos tratamentos odontológicos de rotina (Ioris \& Bacchi, 2019). Outras classes podem ser empregadas a depender da indicação clínica, de acordo com o tratamento odontológico e área de especialização, a exemplo dos benzodiazepínicos, antidepressivos tricíclicos, corticosteroides, analgésicos opioides e os relaxantes musculares (CRO-SP, 2018).

Com o crescimento do número de pacientes polifarmácias, em especial, aqueles que são acometidos por cardiopatias, é necessário que os profissionais da saúde estejam atentos às drogas que são utilizadas por estes pacientes para o controle de suas comorbidades e que possam sofrer interações com aquelas prescritas na rotina de outros tratamentos. As interações medicamentosas são classificadas quanto ao perfil, podendo afetar a farmacodinâmica (quando interfere no mecanismo de ação do fármaco) ou a farmacocinética (interferências na absorção, distribuição, metabolismo e a excreção). Também são classificadas em grave (quando há risco de morte ou necessita da intervenção para evitar a evolução para o estado de saúde grave), moderada (tem-se o risco de agravamento da condição do paciente ou precisa da alteração na terapia) e menor (quando os efeitos clínicos não precisam de mudanças terapêuticas) (Cavalcante, 2018).

No dia-a-dia, os cirurgiões-dentistas, assim como os acadêmicos, que atuam nas clínicas odontológicas dos cursos de Odontologia, deparam-se com pacientes que possuem sintomatologia dolorosa, infecções e ansiedade. Devido ao número expressivo de pacientes hipertensos, sobretudo, utilizando diversas classes anti-hipertensivas, faz-se necessária a disciplina de se observar as interações medicamentosas que podem ocorrer junto ao desenvolvimento dos tratamentos odontológicos (Bertollo et al., 2013; Barroso et al., 2021).

No quadro abaixo, encontram-se as interações medicamentosas e seus possíveis efeitos entre as medicações indicadas para as terapias anti-hipertensivas preconizadas pelas diretrizes da Sociedade Brasileira de Cardiologia em comparação com os fármacos indicados nos tratamentos odontológicos, seguindo as recomendações do guia prático para prescrição e dispensação de medicamentos na Odontologia desenvolvido pelo Conselho Regional de Odontologia de São Paulo. As interações foram pesquisadas junto ao bulário eletrônico da Agência de Vigilância Sanitária (ANVISA, 2020) entre os períodos de agosto de 2020 a fevereiro de 2021 (SBC, 2016; CRO-SP, 2018; Barroso et al., 2021).

Quadro 2. Interações medicamentosas entre os fármacos empregados na terapia anti-hipertensiva e nos tratamentos odontológicos.

\begin{tabular}{|c|c|c|c|c|}
\hline \multirow{2}{*}{$\begin{array}{l}\text { Medicamentos } \\
\text { empregados nos } \\
\text { tratamentos } \\
\text { odontológicos }\end{array}$} & \multicolumn{4}{|c|}{ Possíveis efeitos das interações medicamentosas } \\
\hline & $\begin{array}{l}\text { Classes/Medicações } \\
\text { anti-hipertensivas }\end{array}$ & $\begin{array}{c}\text { Efeitos em } \\
\text { comum }\end{array}$ & $\begin{array}{c}\text { Classes/Medicações } \\
\text { anti-hipertensivas }\end{array}$ & Efeitos Específicos \\
\hline \multirow{3}{*}{ AINES } & \multirow{3}{*}{$\begin{array}{c}\text { Diuréticos tiazídicos: Clortalidona, } \\
\text { Hidroclorotiazida, Indapamida; } \\
\text { Diuréticos de alça: Furosemida, Bumetanida } \\
\left.\text { (Burinax }{ }^{\circledR}\right) ; \\
\text { Poupadores de potássio: Amilorida; } \\
\text { Agentes alfa-agonistas de ação central: } \\
\text { Clonidina; } \\
\text { Bloqueadores de canais cálcio: Nitrendipino, } \\
\text { Diltiazem; } \\
\text { IECA: Captopril (em especial com a } \\
\text { indometacina e o ácido acetilsalicílico), } \\
\text { Enalapril, Lisinopril (em especial com a }\end{array}$} & \multirow{3}{*}{$\begin{array}{l}\text { Diminuição do } \\
\text { efeito anti- } \\
\text { hipertensivo }\end{array}$} & $\begin{array}{l}\text { Diuréticos de alça: } \\
\text { Furosemida (em } \\
\text { especial com os } \\
\text { salicilatos); } \\
\end{array}$ & $\begin{array}{l}\text { Pode provocar o } \\
\text { aumento } \\
\text { plasmático do } \\
\text { diurético. } \\
\end{array}$ \\
\hline & & & $\begin{array}{c}\text { Poupadores de } \\
\text { potássio: Amilorida, } \\
\text { Espironolactona (em } \\
\text { especial a } \\
\text { indometacina e os } \\
\text { salicilatos); }\end{array}$ & $\begin{array}{c}\text { Diminuição do efeito } \\
\text { diurético e } \\
\text { natriurético }\end{array}$ \\
\hline & & & $\begin{array}{l}\text { Bloqueadores de } \\
\text { canais cálcio: } \\
\text { Nitrendipino; }\end{array}$ & $\begin{array}{c}\text { Pode gerar } \\
\text { hemorragias } \\
\text { gastrointestinais }\end{array}$ \\
\hline
\end{tabular}




\begin{tabular}{|c|c|c|c|c|}
\hline & $\begin{array}{l}\text { indometacina), Ramipril, Trandolapril } \\
\left.\text { (Gopten }{ }^{\circledR}\right) ; \\
\text { Bloqueadores dos receptores AT1 da } \\
\text { angiotensina II: Losartana (em especial com a } \\
\text { indometacina e os inibidores seletivos da }\end{array}$ & & $\begin{array}{l}\text { Bloqueadores de } \\
\text { canais cálcio: } \\
\text { Verapamil (em } \\
\text { especial com o ácido } \\
\text { acetilsalicílico); } \\
\end{array}$ & $\begin{array}{l}\text { Pode aumentar os } \\
\text { riscos de } \\
\text { sangramentos }\end{array}$ \\
\hline & $\begin{array}{c}\text { COX-2), Valsartana (em especial aos } \\
\text { inibidores seletivos da COX-2); } \\
\text { Betabloqueadores: Carvedilol, Nadolol } \\
\left.\text { (Corgard }{ }^{\circledR}\right) \text {, Metoprolol (em especial com a } \\
\left.\text { indometacina), Pindolol (Visken }{ }^{\circledR}\right), \\
\text { Propanolol (em especial com indometacina); } \\
\text { Inibidores diretos da renina: Alisquireno; }\end{array}$ & & \multirow{2}{*}{$\begin{array}{l}\text { Betabloqueadores: } \\
\text { Metoprolol (em } \\
\text { especial com anti- } \\
\text { inflamatórios } \\
\text { seletivos a COX-2; }\end{array}$} & \multirow{2}{*}{$\begin{array}{l}\text { Pode elevar os níveis } \\
\text { plasmáticos do anti- } \\
\text { hipertensivo }\end{array}$} \\
\hline & $\begin{array}{l}\text { Diuréticos tiazídicos: Clortalidona, } \\
\text { Indapamida (riscos de insuficiência renal } \\
\text { crônica em pacientes desidratados); } \\
\text { Diuréticos de alça: Furosemida (riscos de } \\
\text { insuficiência renal crônica); } \\
\text { Poupadores de potássio: Amilorida; } \\
\text { IECA: Enalapril, Lisinopril, Ramipril; } \\
\text { Inibidores diretos da renina: Alisquireno; }\end{array}$ & $\begin{array}{l}\text { Riscos a função } \\
\text { renal }\end{array}$ & & \\
\hline \multirow[b]{2}{*}{ Corticosteroides } & $\begin{array}{c}\text { Diuréticos tiazídicos: Clortalidona, } \\
\text { Hidroclorotiazida; } \\
\text { Diuréticos de alça: Furosemida; } \\
\text { Poupadores de potássio: Amilorida; }\end{array}$ & $\begin{array}{l}\text { Aumento do } \\
\text { efeito } \\
\text { hipocalêmico }\end{array}$ & \multirow[b]{2}{*}{ IECA: Ramipril; } & \multirow[b]{2}{*}{$\begin{array}{l}\text { Pode aumentar a } \\
\text { ocorrência de } \\
\text { reações } \\
\text { hematológicas }\end{array}$} \\
\hline & $\begin{array}{c}\text { Diuréticos tiazídicos: Indapamida (deve-se a } \\
\text { maior retenção de sódio); } \\
\text { Agentes alfa-agonistas de ação central: } \\
\left.\text { Rilmenidina (Hymperium }{ }^{\circledR}\right) ; \\
\text { Bloqueadores de canais de cálcio: } \\
\text { Lecarnidipino; } \\
\end{array}$ & $\begin{array}{l}\text { Diminuição do } \\
\text { efeito anti- } \\
\text { hipertensivo }\end{array}$ & & \\
\hline \multirow{4}{*}{ Antibacterianos } & \multirow[b]{2}{*}{$\begin{array}{l}\text { Diuréticos de alça: Furosemida, Bumetanida } \\
\qquad\left(\text { Burinax }^{\circledR}\right) ;\end{array}$} & \multirow{2}{*}{$\begin{array}{l}\text { Com os } \\
\text { aminoglicosídeos: } \\
\text { aumento da } \\
\text { ototoxicidade }\end{array}$} & $\begin{array}{l}\text { Diuréticos } \\
\text { tiazídicos: } \\
\text { Clortalidona; }\end{array}$ & $\begin{array}{l}\text { Com as penicilinas } \\
\text { (em altas doses): } \\
\text { Efeito hipocalêmico }\end{array}$ \\
\hline & & & $\begin{array}{l}\text { Agentes alfa- } \\
\text { agonistas de ação } \\
\text { central: Rilmenidina } \\
\left.\text { (Hymperium }{ }^{\circledR}\right) ;\end{array}$ & $\begin{array}{l}\text { Com a Eritromicina: } \\
\text { aumento do risco de } \\
\text { arritmias }\end{array}$ \\
\hline & \multirow[b]{2}{*}{ Betabloqueadores: Metoprolol, Propanol; } & \multirow{2}{*}{$\begin{array}{l}\text { Com a } \\
\text { Rifampicina: } \\
\text { alterações } \\
\text { plasmáticas dos } \\
\text { anti- } \\
\text { hipertensivos }\end{array}$} & $\begin{array}{l}\text { Bloqueadores dos } \\
\text { receptores AT1 da } \\
\text { angiotensina II: } \\
\text { Valsartana; } \\
\end{array}$ & $\begin{array}{l}\text { Com a Rifampicina: } \\
\text { potencialização do } \\
\text { efeito hipotensor }\end{array}$ \\
\hline & & & $\begin{array}{l}\text { Betabloqueadores: } \\
\text { Carvedilol; }\end{array}$ & $\begin{array}{l}\text { Com a Rifampicina: } \\
\text { diminuição do } \\
\text { controle da pressão } \\
\text { sistólica }\end{array}$ \\
\hline \multirow{4}{*}{$\begin{array}{l}\text { Antidepressivos } \\
\text { tricíclicos }\end{array}$} & \multirow{2}{*}{$\begin{array}{l}\text { Diuréticos tiazídicos: Clortalidona (para } \\
\text { qualquer classe de antidepressivo), } \\
\text { Hidroclorotiazida, Indapamida; } \\
\text { IECA: Trandolapril (Gopten }{ }^{\circledR} \text { ); }\end{array}$} & \multirow[t]{2}{*}{$\begin{array}{l}\text { Potencialização } \\
\text { da hipotensão } \\
\text { ortostática }\end{array}$} & $\begin{array}{l}\text { Diuréticos } \\
\text { tiazídicos: } \\
\text { Clortalidona (para } \\
\text { qualquer classe de } \\
\text { antidepressivo); } \\
\end{array}$ & $\begin{array}{l}\text { Pode provocar o } \\
\text { quadro de } \\
\text { hiponatremia }\end{array}$ \\
\hline & & & $\begin{array}{c}\text { Agentes alfa- } \\
\text { agonistas de ação } \\
\text { central: Clonidina; } \\
\end{array}$ & $\begin{array}{l}\text { Abolição do efeito do } \\
\text { anti-hipertensivo }\end{array}$ \\
\hline & $\begin{array}{l}\text { Vasodilatadores diretos: Hidralazina; } \\
\text { Bloqueadores de canais de cálcio: } \\
\text { Lecarnidipino; } \\
\text { Bloqueadores dos receptores AT1 da } \\
\text { angiotensina II: Losartana; } \\
\text { Betabloqueadores: Nebivolol; }\end{array}$ & $\begin{array}{l}\text { Potencialização } \\
\text { do efeito } \\
\text { hipotensor }\end{array}$ & $\begin{array}{l}\text { Agentes alfa- } \\
\text { agonistas de ação } \\
\text { central: Clonidina; }\end{array}$ & $\begin{array}{l}\text { Abolição do efeito do } \\
\text { anti-hipertensivo }\end{array}$ \\
\hline & $\begin{array}{l}\text { Agentes alfa-agonistas de ação central: } \\
\left.\text { Moxonidina (Cynt }{ }^{\circledR}\right) \text {, Rilmenidina } \\
\left.\text { (Hymperium }{ }^{\circledR}\right) \text {; }\end{array}$ & $\begin{array}{l}\text { Diminuição do } \\
\text { efeito anti- } \\
\text { hipertensivo } \\
\end{array}$ & $\begin{array}{l}\text { Betabloqueadores: } \\
\text { Metoprolol; }\end{array}$ & $\begin{array}{l}\text { Aumento dos níveis } \\
\text { plasmáticos do anti- } \\
\text { hipertensivo }\end{array}$ \\
\hline \multirow{2}{*}{ Anestésicos } & \multirow{2}{*}{$\begin{array}{l}\text { IECA: Trandolapril (Gopten }{ }^{\circledR} \text { ) - com os } \\
\quad \text { anestésicos inalatórios; } \\
\text { Betabloqueadores: Carvedilol, Nebivolol; }\end{array}$} & \multirow{2}{*}{$\begin{array}{l}\text { Potencialização } \\
\text { do efeito } \\
\text { hipotensor }\end{array}$} & $\begin{array}{l}\text { Betabloqueadores: } \\
\text { Nebivolol; }\end{array}$ & $\begin{array}{l}\text { Atenuação da } \\
\text { taquicardia reflexa }\end{array}$ \\
\hline & & & $\begin{array}{l}\text { Betabloqueadores: } \\
\text { Carvedilol; }\end{array}$ & $\begin{array}{l}\text { Poderá ocorrer } \\
\text { efeitos inotrópicos } \\
\text { negativos }\end{array}$ \\
\hline
\end{tabular}




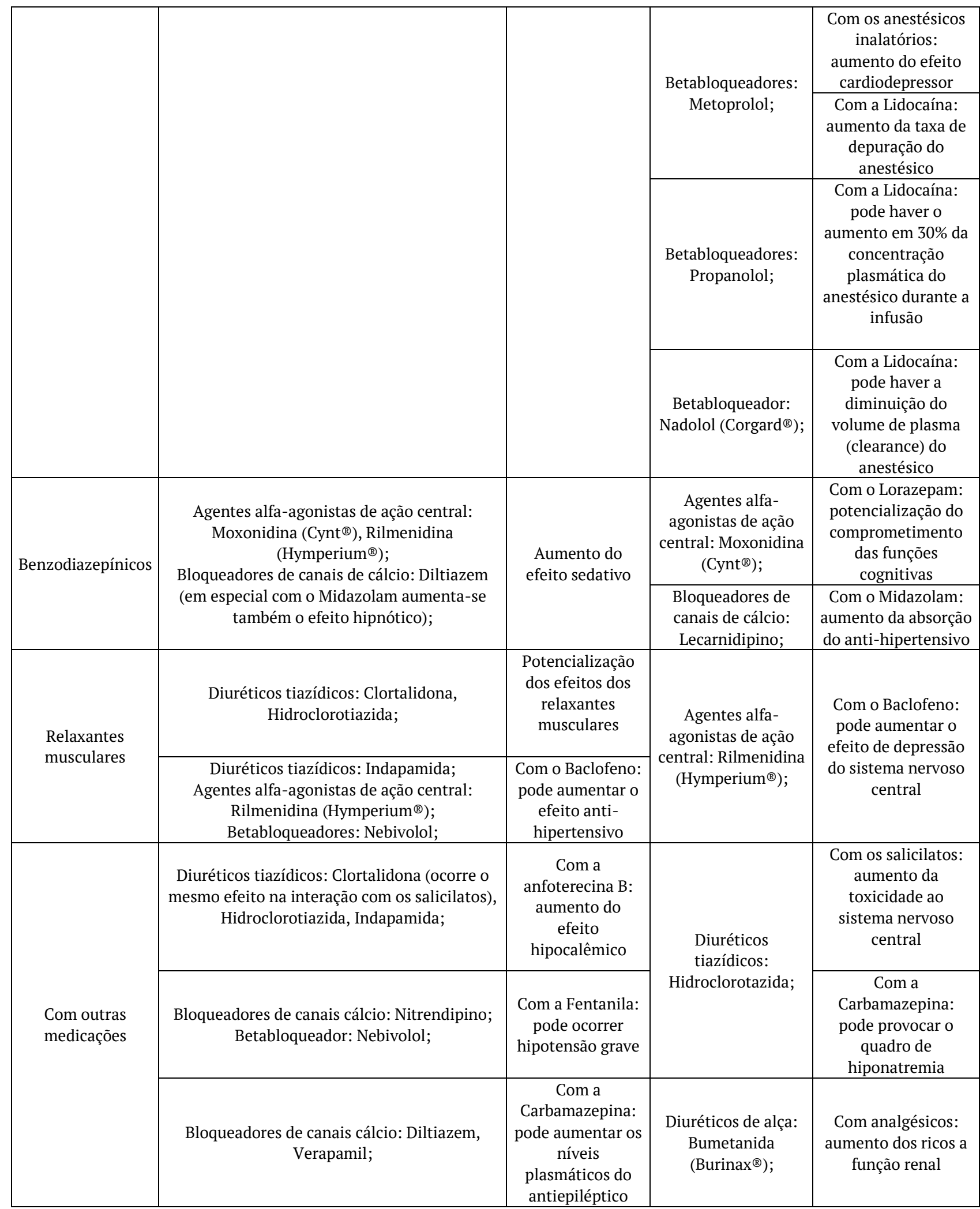

\section{Considerações finais}

Diante da revisão de literatura apresentada, é possível observar a necessidade de precaução no emprego dos medicamentos necessários aos tratamentos odontológicos em conjunto com aqueles aplicados nas terapias anti-hipertensivas, para que assim sejam evitadas interações entre os fármacos. Em especial, chamase a atenção para o uso dos AINES, os quais representam uma das classes mais prescritas na Odontologia, sendo um importante aliado no controle dos processos inflamatórios. A sua utilização requer cautela quando relacionado a boa parte dos medicamentos estabelecidos para o controle da hipertensão arterial, devido a possibilidade de redução do efeito anti-hipertensivo, podendo causar prejuízos aos pacientes. 
Desta forma, a apresentação desta revisão se faz importante para a comunidade acadêmica, tendo em vista que poderá servir de recurso para consulta das possíveis interações medicamentosas no atendimento odontológico dos pacientes hipertensos.

\section{Referências}

Andrade, E. D. 2014. Terapêutica medicamentosa em odontologia. $3^{\text {a }}$ ed. São Paulo: Editora Artes Médicas LTD.

ANVISA. Agência Nacional de Vigilância Sanitária. 2020. Bulário eletrônico [Versão Eletrônica]. Disponível em: <https://consultas.anvisa.gov.br/\#/bulario/>. Acesso em: 28 out. 2020.

Barroso, W. K. S., Rodrigues, C. I. S., Bortolotto, L. A., Mota-Gomes, M. A., Brandão, A. A., \& Nadruz, W. 2021. Diretrizes Brasileiras de Hipertensão Arterial - 2020. Arquivos Brasileiros de Cardiologia, 116(3), 516-658.

Bertollo, A. L., Demartini, C., \& Piato, A. L. 2013. Interações medicamentosas na clínica odontológica. Revista Brasileira de Odontologia, 70(2), 120-124.

Bombig, M. T. N., \& Póvoa, R. 2009. Interações e associações de medicamentos no tratamento antihipertensivo - Antagonistas dos canais de cálcio. Revista Brasileira de Hipertensão, 16(4), 226-230.

Cavalcante, A. L. S. 2018. Interações medicamentosas potenciais em pacientes cardiopatas admitidos em um hospital universitário. Trabalho de conclusão de curso (Graduação em Farmácia), Faculdade de Ceilândia, Universidade de Brasília, Brasília.

CRO-SP. Conselho Regional de Odontologia de São Paulo. 2018. Guia Prático de prescrição e dispensação de medicamentos na Odontologia. Disponível em: <http://www.crosp.org.br/uploads/arquivo/e1b1db518290aaef2175d1ac72f7a631.pdf>. Acesso em: 20 ago 2020.

Haese, R. D. P., \& Cançado, R. P. 2016. Urgências e emergências médicas em odontologia: avaliação da capacitação e estrutura dos consultórios de cirurgiões-dentistas. Revista de Cirurgia e Traumatologia BucoMaxilo-Facial, 16(3), 31-39.

Hall, J. E. 2017. Tratado de Fisiologia Médica. 13a ed. Rio de Janeiro: Elsevier.

Ioris, L. M., \& Bacchi, A. 2019. Interações medicamentosas de interesse em odontologia. Revista da Faculdade de Odontologia, 24(1), 148-154.

Little, J.W., Falace, D. A., Miller, C. S., \& Rhodus, N. L. 2009. Manejo odontológico do paciente clinicamente comprometidos. $7^{\text {a }}$ ed. Rio de Janeiro: Elsevier.

Matos, J. D. M., Pereira, A. L. C., Lopes, G. R. S., Andrade, V. C., \& Perez, E. G. 2018. Comportamento da pressão arterial sistêmica em pacientes submetidos a procedimentos cirúrgicos odontológicos. Revista da Faculdade de Odontologia, 23(3), 361-370.

Nascimento, R. C. R. M., Álvares, J., Júnior, A. A. G., Gomes, I. C., Silveira, M. R., Costa, E.A., \& Acurcio, F. A. 2017. Polifarmácia: uma realidade na atenção primária do Sistema Único de Saúde. Revista de Saúde Pública, 51(suppl 2), 19s.

Ritter, J. M., Flower, R., Henderson, G., Loke, Y. K., MacEwan, D., \& Rang, H. P. 2020. Rang \& Dale: farmacologia. $9^{\mathrm{a}}$ ed. Rio de Janeiro: Guanabara Koogan.

Scolari, F. L., Leitão, S. A. T., Faganello, L. S., Goldraich, L.A., \& Clausell, N. 2018. Insuficiência cardíaca fisiopatologia atual e implicações terapêuticas. Revista da Sociedade de Cardiologia do Estado de São Paulo, 28(1), 33-41.

SBC. Sociedade Brasileira de Cardiologia. 2016. $7^{\text {a }}$ Diretriz Brasileira de Hipertensão Arterial. Arquivos Brasileiros de Cardiologia, 107(3), 1-83.

\section{Minicurrículo}

Saulo Rodrigues dos Santos. Acadêmico de Odontologia da Universidade Federal de Sergipe (UFS), Campus Aracaju. Membro fundador da Liga Acadêmica de Cirurgia Oral Menor de Sergipe - LACOSE. Foi monitor da disciplina Elementos de Anatomia Humana do Departamento de Morfologia da UFS- Campus São Cristóvão. 
André Luis Silva Santos. Acadêmico de Odontologia da Universidade Federal de Sergipe, Campus Aracaju. Membro fundador da Liga Acadêmica de Cirurgia Oral Menor de Sergipe - LACOSE. Monitor da disciplina de Cirurgia I do Departamento de Odontologia da UFS, campus Aracaju.

Hélio Igor Melo de Albuquerque. Possui graduação em Odontologia pela Universidade Estadual da Paraíba (2005). Residência em Cirurgia e Traumatologia Bucomaxilofacial pelo Hospital de Base do Distrito Federal. Mestre em Odontologia pela Universidade Federal de Sergipe (2020). Foi Professor Voluntário Disciplina de Clínica Integrada I da Faculdade de Odontologia da Universidade Federal de Sergipe. Atualmente é Cirurgião Bucomaxilofacial do Hospital de Urgência de Sergipe.

Luiz Carlos Ferreira da Silva. Possui graduação em Odontologia pela Universidade Federal de Sergipe (1989), Mestrado em Patologia Oral pela Universidade Federal do Rio Grande do Norte (2000), Doutorado em Cirurgia Buco-Maxilo-Facial pela Universidade de Pernambuco (2006) e Fellow em Cirurgia Maxilo-Facial pela Duke University (USA). É Professor Associado de Cirurgia Buco-Maxilo-Facial da Universidade Federal de Sergipe, Coordenador do Serviço de Cirurgia Buco-Maxilo-Facial do Hospital Primavera e atende em clínica privada (Sorcise) e no Hospital da Polícia Militar. Tem prática exclusiva em Cirurgia Buco-Maxilo-Facial, atuando principalmente nos seguintes temas: patologia oral, terceiros molares, cirurgia ortognática e implantes.

Como citar: Santos, S.R., Santos, A.L.S., Albuquerque, H.I.M., \& Silva, L.C.F. 2022. Interações medicamentosas entre terapias anti-hipertensivas e terapêutica odontológica: revisão de literatura. Pubsaúde, 8, a271. DOI: https://dx.doi.org/10.31533/pubsaude8.a271

Recebido: 30 out. 2021.

Revisado e aceito: 04 nov. 2021.

Conflito de interesse: os autores declaram, em relação aos produtos e companhias descritos nesse artigo, não ter interesses associativos, comerciais, de propriedade ou financeiros que representem conflito de interesse.

Licenciamento: Este artigo é publicado na modalidade Acesso Aberto sob a licença Creative Commons Atribuição 4.0 (CC-BY 4.0). 